\title{
Estudio de la prevalencia de trastornos ansiosos y depresivos en pacientes en hemodiálisis
}

\author{
Eutropio Moreno Núñez** \\ $M^{a}$ Dolores Arenas Jiménez(*) \\ Esperanza Porta Bellmar** \\ Laura Escalant Calpena* \\ $M^{a}$ José Cantó García* \\ Gema Castell García* * \\ Felipe Serrano Gómez* \\ Javier Samper Llobregat* * * \\ Diana Millán Mayol* \\ José M. Cases Iborra**
}

Nefrólogo(*)

Hospital P. Socorro de Alicante*

Hospital General U. de Alicante**

Hospital P. Socorro de Elche***

\section{RESUMEN}

Introducción y objetivos: La vida de los pacientes en hemodiálisis está sometida a diferentes situaciones estresantes y amenazantes. Esto hace que un porcentaje nada despreciable de pacientes presente trastornos ansiosos y depresivos como respuesta normal a estas situaciones que pueden pasar desapercibidos al personal de salud que les atiende. Por otra parte, estos trastornos afectivos pueden influir en la percepción de su propio estado de salud. El objetivo del trabajo es conocer la prevalencia de estos trastornos ansiosos y depresivos en nuestros pacientes hemodializados y ver su influencia en la calidad de vida (CVRS).

Metodología: Se realiza el estudio con 75 pacientes, 50 varones. Se excluyeron los pacientes mayores de 65 años y con menos de 6 meses de permanencia en programa de $\mathrm{HD}$, o que presentaran trastornos psicóticos, neurológicos o descompensación del estado físico. La depresión se ha medido

\begin{tabular}{|c|}
\hline Correspondencia: \\
Eutropio Moreno Núñez \\
Unidad de Hemodiálisis \\
Hospital G. Universitario de Alicante \\
c/ Maestro Alonso 109 \\
03010 Alicante
\end{tabular}

con dos escalas: Inventario de Beck (BDI) y la Escala de Hamilton (HDRS). La ansiedad se midió con la Escala de Hamilton para ansiedad (HARS) y la CVRS con la versión española de las láminas COOP/WONCA.

Resultados: La sintomatología depresiva estaba presente en un porcentaje bastante elevado de nuestros pacientes: $53,3 \%$ con la HDRS y un $44 \%$ con el BDI y la ansiosa en un 46,6\%. Al analizar los resultados observamos que las variables sociodemográficas, así como las relacionadas con el proceso dialítico no han resultado factores predisponentes de la ansiedad y la depresión. Encontramos un buen nivel de correlación (C. de Pearson) entre la CVRS medida con el COOP/WONCA y los estados de ansiedad $(r=0,52$ (HARS), $p<0,001)$ y depresión $(r=$ 0,64 (BDI) y 0,75 (HDRS); $p<0,001$ ).

Conclusiones: Existe una elevada prevalencia de sintomatología ansiosa y depresiva en nuestros pacientes asociada a factores relacionados con la CVRS y su capacidad funcional.

PALABRAS CLAVES: HEMODIÁLISIS

ANSIEDAD

DEPRESIÓN

CALIDAD DE VIDA (CVRS) 


\section{STUDY OF THE PREVALENCE OF ANXIOUS AND DEPRESSIVE DISORDERS IN PATIENTS UNDER HAEMODIALYSIS}

\section{ABSTRACT}

Introduction and objectives: The lives of patients under haemodialysis are subject to different stressful, threatening situations. This means that a not inconsiderable percentage of patients suffer anxious and depressive disorders as a normal reaction to such situations, which might be unnoticed by the health personnel attending them. Furthermore, these affective disorders may influence the perception of their state of health. The aim of the work is to discover the prevalence of these anxious and depressive disorders in our patients under haemodialysis and see the influence on their quality of life (CVRS).

Methodology: The study was carried out with 75 patients, 50 of whom were men. Patients over 65 years of age were excluded and also those having been on an HD programme for under 6 months, or those presenting psychotic, neurological disorders or decompensation of their physical state. The depression was measured with two scales: Beck Inventory (BDI) and the Hamilton Scale (HDRS). The anxiety was measured with the Hamilton Scale for anxiety (HARS) and the CVRS with the Spanish version of the COOP/WONCA laminas.

Results: Symptoms of depression were present in a fairly high percentage of our patients: $53.3 \%$ with HDRS and $44 \%$ with BDI and $46.6 \%$ in the anxious side. On analysing the results, we observed that the sociodemographic variables, and those related to the dialysis process, were not predisposing factors of anxiety and depression. We find a good correlation (Pearson C.) between the CVRS measured with the COOP/WONCA and the states of anxiety $(r=0.52$ (HARS), $\mathrm{p}<0.001)$ and depression $(\mathrm{r}=0.64$ (BDI) and 0.75 (HDRS); $\mathrm{p}<0.001$ ).

Conclusions: There is a large prevalence of symptoms of anxiety and depression among our patients, associated with factors related to the CVRS and their functional capacity.

KEYWORDS:

HAEMODIALYSIS
ANXIETY
DEPRESSION
QUALITY OF LIFE (CVRS)

\section{INTRODUCCIÓN}

Todos los pacientes en hemodiálisis se encuentran dependientes de una máquina, un centro y un personal sanitario del que depende su vida, además de estar expuestos a otros factores de estrés como el turno, un transporte o una dieta. Todo se complica con otros problemas como el fin de la diuresis, trastornos en su función sexual y en general, la presencia constante de situaciones amenazantes a las que se tienen que enfrentar estos enfermos. Los trastornos ansiosos y depresivos pueden ser respuestas normales a todas estas manifestaciones ${ }^{1-2}$.

La ansiedad es un estado afectivo de temor, inseguridad, tensión o alerta. Es un afecto que mira al presente y al futuro. La depresión, al contrario, es la consecuencia de experiencias que tienen que ver con el pasado, normalmente con pérdidas. La presencia de ansiedad y depresión no es, por si misma, síntoma de trastorno mental alguno, lo que si puede ser anómalo es cuando su intensidad y duración es desproporcionada al estímulo que las desencadena ${ }^{3}$.

La prevalencia de los trastornos afectivos difiere según los diversos autores entre el 25 y el 50\% y algunos llegan hasta el 70\%. Cuando se usan como criterios los diagnósticos del DMSIII (los síntomas tienen que permanecer más tiempo que en el resto de escalas) no pasa del $10 \%$. Los factores que explican tanta variabilidad pueden ser las diferencias en el proceso de selección de los pacientes y los métodos de cribado y diagnóstico utilizados ${ }^{4-5 \cdot 6}$.

Los problemas psicológicos de los enfermos renales no se toman suficiente en cuenta, y no es detectada la manera de como esos problemas influyen en la producción de síntomas corporales y más concretamente en su calidad de vida ${ }^{7}$.

\section{OBJETIVOS}

Conocer la prevalencia de trastornos afectivos y ansiosos en nuestros pacientes hemodializados y su asociación con otras variables sociodemográficas o relacionadas con su tratamiento, así como su influencia en la calidad de vida, con el fin de identificar factores que permitan mejorar su detección para poder establecer una adecuada relación de ayuda al paciente.

\section{METODOLOGÍA}

Diseño: Estudio descriptivo, transversal, observacional. Población: La muestra del estudio la componen 75 pacientes: 50 hombres y 25 mujeres. Todos ellos han par- 
ticipado de forma voluntaria y han dado su consentimiento informado.

Criterios de inclusión: La muestra se obtuvo de todos lo pacientes en tratamiento en hemodiálisis con más de seis meses en programa con edades comprendidas entre 18 y 65 años (los mayores de 65 años pueden puntuar positivo en escalas de ansiedad y depresión en caso de padecer un cuadro de deterioro cognitivo. Se analizaran en un estudio posterior con las escalas apropiadas), durante el periodo comprendido entre diciembre del 2002 y enero del 2003.

Criterios de exclusión: Se excluyeron los pacientes previamente diagnosticados de trastorno psicótico, trastorno neurológico, retraso mental, que hubieran padecido una descompensación en su estado físico y la presencia de un acontecimiento vital estresante en los últimos 30 días.

\section{Variables:}

- Sociodemográficas: Edad, sexo, estado civil, nivel de estudios, situación laboral

- Relacionadas con el proceso dialítico: Tiempo en HD, tiempo sesión de $\mathrm{HD}$, ganancia de peso interdiálisis, $\mathrm{n}$ o de fármacos, cumplimiento terapéutico, hemoglobina, fósforo, potasio y KTV.

- Variables psicosociales: Aislamiento social, depresión, ansiedad y valoración funcional y de calidad de vida

\section{Test y escalas de valoración utilizadas:}

- Inventario de Depresión de Beck (BDI $)^{8}$ : Es una escala autoaplicada de 21 ítems concebidos para la detección y cuantificación del síndrome depresivo en adultos. Se le pide al paciente que en cada uno de los 21 apartados marque con una cruz a la izquierda la frase que mejor refleje su situación durante la última semana. El rango de la puntuación obtenida es de 0 a 63. Los puntos de corte que se proponen para graduar el síndrome depresivo son los siguientes:

\section{0 a 9 Ausente \\ 10 a 18 Leve \\ 19 a 29 Moderada 30 a 63 Grave}

- Escala de Hamilton para la Depresión (HDRS): Consta de 17 ítems heteroaplicados que evalúan el perfil sintomatológico y la gravedad del cuadro depresivo. El marco de referencia temporal es el de los últimos días. Para cada ítem la escala proporciona criterios operativos de puntuación. La escala proporciona una puntuación global de gravedad del cuadro y se ha interpretado de la siguiente manera:

\section{O a 7 No Depresión \\ 8 a 14 Depresión leve \\ $>$ de 15 Depresión moderada o grave}

- Escala de Hamilton para la Ansiedad (HARS) ${ }^{10}$. Es una escala heteroaplicada que valora la intensidad de la ansiedad. Consta de 14 ítems que evalúan los aspectos físicos, psíquicos y conductuales de la ansiedad. El marco de referencia temporal es los últimos días en todos los ítems, excepto en el último en el que se valora la conducta del sujeto durante la entrevista. Los puntos de corte recomendados sobre un total de 56 son:

\section{0 a 5 No Ansiedad \\ 6 a 14 Ansiedad leve \\ $>15$ Ansiedad moderada o grave}

- Láminas de COOP/WONCA ${ }^{11}$ : Es un instrumento diseñado para medir la calidad de vida relacionada con la salud de un modo rápido, sencillo, comprensible y breve. El cuestionario comprende 9 escalas distintas y con un único ítem (figura 1). Cada una de las escalas presenta un título y plantea una cuestión que tiene que ver con lo sucedido durante las dos últimas semanas. A estas cuestiones, se contesta con una de las 5 posibles alternativas de respuesta. Los títulos de las escalas son: forma física, sentimientos, actividades cotidianas, actividades sociales, cambio en el estado de salud, estado de salud, dolor, apoyo social y calidad de vida.

Recogida de datos: Se ha diseñado un cuaderno de recogida de datos para cada paciente que incluye las variables comentadas en el apartado anterior y los resultados de las escalas pasadas a los pacientes. Con el fin mejorar la uniformidad en la recogida de datos, se consensuó entre los entrevistadores el modo de solicitar la colaboración del paciente y hacer las encuestas.

Procedimientos estadísticos/análisis de los resultados: Los resultados fueron procesados por el programa EXCEL Microsoft 2000 sobre el que se elaboró la base de datos y fueron analizados por el paquete estadístico SPSS11 WIN. Se utilizaron Chi cuadrado, Correlación de Pearson y Spearman para el análisis de las variables cualitativas y numéricas según procediese.

\section{RESULTADOS}

En las tablas 1,2, se muestran los resultados descriptivos de los pacientes y variables a estudio. 
Tabla 1

\begin{tabular}{|c|c|c|}
\hline \multicolumn{3}{|c|}{$\begin{array}{l}\text { Variables sociodemográficas de la población } \\
\qquad \text { en estudio }\end{array}$} \\
\hline & Frecuencia & Porcentaje \\
\hline \multicolumn{3}{|l|}{$\begin{array}{l}\text { Total pacientes } \\
\text { por centro }\end{array}$} \\
\hline HGU Alicante & 22 & $29,3 \%$ \\
\hline SPS Alicante & 43 & $57,3 \%$ \\
\hline SPS Elche & 10 & $13,3 \%$ \\
\hline \multicolumn{3}{|l|}{ Edad } \\
\hline $18-35$ & 11 & $14,7 \%$ \\
\hline $36-50$ & 25 & $33,3 \%$ \\
\hline $51-65$ & 39 & $52 \%$ \\
\hline \multicolumn{3}{|l|}{ Sexo } \\
\hline Mujeres & 25 & $33,3 \%$ \\
\hline Hombres & 50 & $66,7 \%$ \\
\hline \multicolumn{3}{|l|}{ Estado civil } \\
\hline Casado & 53 & $70 \%$ \\
\hline Soltero,viudo, divorciado & 22 & $30 \%$ \\
\hline \multicolumn{3}{|l|}{ Nivel de estudios } \\
\hline Primarios & 59 & $78,7 \%$ \\
\hline Secundarios & 13 & $17,3 \%$ \\
\hline Superiores & 3 & $4,0 \%$ \\
\hline \multicolumn{3}{|l|}{ Situación Laboral } \\
\hline Activo & 21 & $28 \%$ \\
\hline Pensionista & 54 & $72 \%$ \\
\hline \multicolumn{3}{|l|}{ Aislamiento social } \\
\hline $\mathrm{Si}$ & 8 & $10,7 \%$ \\
\hline No & 67 & 89,3 \\
\hline
\end{tabular}

Tabla 2

\begin{tabular}{|c|c|c|}
\hline \multicolumn{3}{|c|}{$\begin{array}{l}\text { Variables relacionadas con el } \\
\text { proceso dialítico }\end{array}$} \\
\hline & Frecuencia & Porcentaje \\
\hline Tiempo en HD & 25 & $333 \%$ \\
\hline 2o $332-1410$ meses & 25 & $\begin{array}{l}35,3 \% \\
33,3 \%\end{array}$ \\
\hline $3^{\circ}>$ de 141 meses & 25 & $33,3 \%$ \\
\hline Tiempo sesión HD & & \\
\hline$\leq 3,5$ Horas & 44 & $58,7 \%$ \\
\hline 4 Horas & 31 & $41,3 \%$ \\
\hline $\begin{array}{l}\text { Transporte a } \\
\text { centro HD }\end{array}$ & & \\
\hline Propio & 45 & $60 \%$ \\
\hline Comunitario & 50 & $40 \%$ \\
\hline Diuresis residual & & \\
\hline & 14 & $18,7 \%$ \\
\hline No & 61 & $81,3 \%$ \\
\hline Programa trasplante & & \\
\hline Incluidos & 29 & $38,7 \%$ \\
\hline Excluidos & 46 & $61,3 \%$ \\
\hline Farmacos & & \\
\hline $\begin{array}{l}\leq \text { de } 6 \\
\text { de } 7\end{array}$ & $\begin{array}{l}26 \\
49\end{array}$ & $\begin{array}{l}34,7 \% \\
65,3 \%\end{array}$ \\
\hline $\begin{array}{l}\text { Cumplimiento } \\
\text { terapeútico }\end{array}$ & & \\
\hline$\leq \mathrm{del} 80 \%$ & 58 & $77,3 \%$ \\
\hline$<$ del $80 \%$ & 17 & $22,7 \%$ \\
\hline
\end{tabular}

En la tabla 3 se muestran los resultados de la sintomatología ansiosa y depresiva en nuestros pacientes de hemodiálisis.

\section{Tabla 3}

Sintomatología ansiosa y depresiva según las escalas utilizadas

Sint. depresiva según la Escala de Hamilton (HDRS)

\section{Frecuencia}

Porcentaje

Ausente

Leve

Moderada o grave

\section{5}

23

17

\section{Sint. depresiva según el Inventario de Beck (BDI)}

Ausente

Leve

Moderada

Severa

Sint: ansiosa según la Escala de Hamilton (HARS)

Ausente

Leve

Moderada

\begin{tabular}{|r|r}
\hline 35 & $46,7 \%$ \\
23 & $30,7 \%$ \\
17 & $22,7 \%$ \\
\hline & \\
42 & $56 \%$ \\
22 & $29,3 \%$ \\
9 & $12 \%$ \\
2 & $2,7 \%$ \\
\hline & \\
40 & $53,3 \%$ \\
10 & $33.3 \%$ \\
\hline
\end{tabular}


En la tabla 4 podemos ver los resultados por pacientes en cada una de las respuestas y la puntuación media en cada una de las láminas COOP/WONCA para evaluar la CVRS.

Tabla 4

\begin{tabular}{|c|c|c|c|c|c|c|c|}
\hline \multicolumn{8}{|c|}{ Resultados de calidad de vida y funcionalidad con las laminas de COOP / WOONCA } \\
\hline $\begin{array}{l}\text { LAMINAS } \\
\text { COOC/WONKA }\end{array}$ & $\begin{array}{l}\text { PUNTUACIÓN } \\
\text { Media x lámina }\end{array}$ & 1 & 2 & $\begin{array}{c}\text { PUNTUACIÓN } \\
3\end{array}$ & 4 & 5 & $\begin{array}{c}\text { TOTAL } \\
\text { PACIENTES }\end{array}$ \\
\hline $\begin{array}{c}\text { Forma física } \\
1\end{array}$ & 3,2 & $\begin{array}{c}\text { Muy intensa } \\
3\end{array}$ & $\begin{array}{c}\text { Intensa } \\
7\end{array}$ & $\begin{array}{c}\text { Moderada } \\
34\end{array}$ & $\begin{array}{l}\text { Ligera } \\
27\end{array}$ & $\begin{array}{c}\text { Muy ligera } \\
4\end{array}$ & 75 \\
\hline $\begin{array}{c}\text { Sentimientos } \\
2\end{array}$ & 2 & $\begin{array}{c}\text { Nada en absolu } \\
30\end{array}$ & $\begin{array}{c}\text { Un poco } \\
23\end{array}$ & $\begin{array}{l}\text { Moderadamente } \\
11\end{array}$ & $\begin{array}{c}\text { Bastante } \\
7\end{array}$ & $\begin{array}{c}\text { Intensamente } \\
4\end{array}$ & 75 \\
\hline $\begin{array}{c}\text { Actv. Cotidian } \\
3\end{array}$ & 1,96 & $\begin{array}{c}\text { No dificultad } \\
34\end{array}$ & $\begin{array}{c}\text { Poca dificultad } \\
24\end{array}$ & $\begin{array}{l}\text { Difi. Moderada } \\
6\end{array}$ & $\begin{array}{c}\text { Much.dificult } \\
8\end{array}$ & $\begin{array}{c}\text { No hacer nada } \\
3\end{array}$ & 75 \\
\hline $\begin{array}{c}\text { Actv. Sociales } \\
4\end{array}$ & 1,7 & $\begin{array}{c}\text { Nada en abslut } \\
50\end{array}$ & $\begin{array}{c}\text { Ligeramente } \\
8\end{array}$ & $\begin{array}{c}\text { Moderadamente } \\
7\end{array}$ & $\begin{array}{c}\text { Bastante } \\
7\end{array}$ & $\begin{array}{c}\text { Muchísimo } \\
3\end{array}$ & 75 \\
\hline $\begin{array}{l}\text { Camb. E. salud } \\
5\end{array}$ & 2,76 & $\begin{array}{c}\text { Mucho mejor } \\
3\end{array}$ & $\begin{array}{c}\text { Un poco mejor } \\
15\end{array}$ & $\begin{array}{l}\text { Igual por estilo } \\
54\end{array}$ & $\begin{array}{c}\text { Un poco peor } \\
3\end{array}$ & $\begin{array}{c}\text { Mucho peor } \\
0\end{array}$ & 75 \\
\hline $\begin{array}{c}\text { Estado salud } \\
6\end{array}$ & 3,24 & $\begin{array}{c}\text { Excelente } \\
2\end{array}$ & $\begin{array}{c}\text { Muy buena } \\
11\end{array}$ & $\begin{array}{c}\text { Buena } \\
33\end{array}$ & $\begin{array}{c}\text { Regular } \\
25\end{array}$ & $\begin{array}{c}\text { Mala } \\
4\end{array}$ & 75 \\
\hline $\begin{array}{l}\text { Dolor } \\
7\end{array}$ & 2,3 & $\begin{array}{c}\text { Nada de dolor } \\
27\end{array}$ & $\begin{array}{c}\text { Dolor muy leve } \\
18\end{array}$ & $\begin{array}{c}\text { Dolor Ligero } \\
12\end{array}$ & $\begin{array}{c}\text { Dolor moderad } \\
13\end{array}$ & $\begin{array}{c}\text { Dolor intenso } \\
5\end{array}$ & 75 \\
\hline $\begin{array}{c}\text { Apoyo social } \\
8\end{array}$ & 2,32 & $\begin{array}{c}\text { Ayuda de todos } \\
23\end{array}$ & $\begin{array}{c}\text { Bastante gente } \\
24\end{array}$ & $\begin{array}{c}\text { Algunas person } \\
14\end{array}$ & $\begin{array}{c}\text { Alguien había } \\
9\end{array}$ & $\begin{array}{c}\text { Nadie en abslut } \\
5\end{array}$ & 75 \\
\hline $\begin{array}{c}\text { Calidad de vida } \\
9\end{array}$ & 2,36 & $\begin{array}{c}\text { Todo muy bien } \\
8\end{array}$ & $\begin{array}{c}\text { Bastante bien } \\
38\end{array}$ & $\begin{array}{c}\text { Bien / Mal } \\
24\end{array}$ & $\begin{array}{c}\text { Bastante mal } \\
4\end{array}$ & $\begin{array}{c}\text { Muy mal } \\
1\end{array}$ & 75 \\
\hline \multicolumn{2}{|c|}{$\begin{array}{c}\text { Puntuación Media Total } \\
\qquad 19,8\end{array}$} & \multicolumn{6}{|c|}{ Excluimos la lámina $n^{\circ} 5$ (cambio estado de salud) por estructura bipolar } \\
\hline
\end{tabular}

En la tabla 5 se recoge el alto nivel de asociación mostrado por las escalas utilizadas en el estudio para evaluar la sintomatología ansiosa y depresiva.

Tabla 5

\begin{tabular}{|c|}
\hline Correlación estadística entre los resultados de las escalas utilizadas \\
\hline \hline ESCALA DEPRESIÓN DE HAMILTON E INDICE PARA DEPRESION DE BECK \\
Correlación de Pearson $=0,704 p<0,001$ \\
\hline ESCALA DEPRESIÓN DE HAMILTON Y ESCALA ANSIEDAD DE HAMILTON \\
Correlación de Pearson $=0,803 p<0,001$ \\
\hline ESCALA ANSIEDAD DE HAMILTON E INDICE PARA DEPRESION DE BECK \\
Correlación de Pearson $=0,578 p<0,001$
\end{tabular}


En la tabla 6 se muestra que menos en la W5 y la W8 existe un buen nivel de correlación entre la salud percibida por el paciente mediante las láminas $\mathrm{C} / \mathrm{W}$ y la sintomatología ansiosa y depresiva.

Tabla 6

Correlación índices ansiosos y depresivos con calidad de vida (COOP/WONCA)

\begin{tabular}{|l|c|c|c|c|c|c|c|c|c|}
\hline \hline & $\begin{array}{c}\text { W1 } \\
\text { F. fisica }\end{array}$ & $\begin{array}{c}\text { W2 } \\
\text { Sentimien }\end{array}$ & $\begin{array}{c}\text { W3 } \\
\text { Act.Coti }\end{array}$ & $\begin{array}{c}\text { W4 } \\
\text { Act.Soci }\end{array}$ & $\begin{array}{c}\text { W5 } \\
\text { Cam.Salud }\end{array}$ & $\begin{array}{c}\text { W6 } \\
\text { Est.Salud }\end{array}$ & $\begin{array}{c}\text { W7 } \\
\text { Dolor }\end{array}$ & $\begin{array}{c}\text { W8 } \\
\text { Ap.Social }\end{array}$ & $\begin{array}{c}\text { W9 } \\
\text { Cal.Vida }\end{array}$ \\
\hline $\begin{array}{l}\text { Depresión } \\
\text { Hamilton }\end{array}$ & $\mathrm{P}<0,004$ & $\mathrm{P}<0,00$ & $\mathrm{P}=0,00$ & $\mathrm{P}<0,000$ & $\mathrm{~N} \mathrm{~S}$ & $\mathrm{P}<000$ & $\mathrm{P}<0,001$ & $\mathrm{~N} \mathrm{~S}$ & $\mathrm{P}<0,000$ \\
\hline $\begin{array}{l}\text { Depresión } \\
\text { I. de Beck }\end{array}$ & $\mathrm{P}<0,000$ & $\mathrm{P}<0,00$ & $\mathrm{P}=0,00$ & $\mathrm{P}<0,000$ & $\mathrm{~N} \mathrm{~S}$ & $\mathrm{P}<002$ & $\mathrm{P}<0,001$ & $\mathrm{~N} \mathrm{~S}$ & $\mathrm{P}<0,000$ \\
\hline $\begin{array}{l}\text { Ansiedad } \\
\text { Hamilton }\end{array}$ & $\mathrm{N} \mathrm{S}$ & $\mathrm{P}<0,00$ & $\mathrm{P}=0,001$ & $\mathrm{P}<0,000$ & $\mathrm{~N} \mathrm{~S}$ & $\mathrm{P}<0,002$ & $\mathrm{P}<0,011$ & $\mathrm{~N} \mathrm{~S}$ & $\mathrm{P}<0,00$ \\
\hline
\end{tabular}

Las tablas 7 y 8 reflejan los resultados y la correlación estadística de la sintomatología ansiosa y depresiva con las distintas variables estudiadas: sociodemográficas y del proceso dialítico. En ambas la correlación resulta negativa en todas las variables estudiadas.

\section{Tabla 7}

\begin{tabular}{|c|c|c|c|c|c|c|c|c|}
\hline \multicolumn{9}{|c|}{ Correlación ansiedad y depresión con variables sociodemográficas } \\
\hline \multirow{2}{*}{$\begin{array}{l}\text { Variables } \\
\text { sociodem }\end{array}$} & \multicolumn{3}{|c|}{ VARIABLES DEPRESIÓN } & \multirow{2}{*}{$\begin{array}{c}\text { Sign. } \\
\text { estadística }\end{array}$} & \multicolumn{3}{|c|}{ VARIABLES ANSIEDAD } & \multirow{2}{*}{$\begin{array}{c}\text { Sign. } \\
\text { estadística }\end{array}$} \\
\hline & AUSENTE & LEVE & MODERADA & & AUSENTE & LEVE & MODERADA & \\
\hline $\begin{array}{l}\text { EDAD } \\
18-35 \\
36-50 \\
51-65 \\
\end{array}$ & $\begin{array}{c}3 \\
12 \\
9 \\
\end{array}$ & $\begin{array}{c} \\
7 \\
9 \\
18 \\
\end{array}$ & $\begin{array}{c}1 \\
1 \\
4 \\
12 \\
\end{array}$ & NS & $\begin{array}{c} \\
4 \\
18 \\
18 \\
\end{array}$ & $\begin{array}{c} \\
6 \\
5 \\
14 \\
\end{array}$ & $\begin{array}{l}1 \\
2 \\
7 \\
\end{array}$ & NS \\
\hline $\begin{array}{l}\text { SEXO } \\
\text { MUJER } \\
\text { HOMBRE }\end{array}$ & $\begin{array}{c}9 \\
26 \\
\end{array}$ & $\begin{array}{c}9 \\
14 \\
\end{array}$ & $\begin{array}{c}7 \\
10 \\
\end{array}$ & NS & $\begin{array}{l}12 \\
28 \\
\end{array}$ & $\begin{array}{c}8 \\
17 \\
\end{array}$ & $\begin{array}{l}5 \\
5 \\
\end{array}$ & NS \\
\hline $\begin{array}{l}\text { E.CIVIL } \\
\text { CASADO } \\
\text { NO CASD }\end{array}$ & $\begin{array}{l}24 \\
11 \\
\end{array}$ & $\begin{array}{l}12 \\
11 \\
\end{array}$ & $\begin{array}{c}17 \\
0\end{array}$ & NS & $\begin{array}{l}27 \\
13 \\
\end{array}$ & $\begin{array}{c}17 \\
8\end{array}$ & $\begin{array}{l}9 \\
1 \\
\end{array}$ & NS \\
\hline $\begin{array}{l}\text { NIV.ESTD } \\
1^{\circ} \\
2^{\underline{a}} \\
\text { Superiores }\end{array}$ & $\begin{array}{c}29 \\
5 \\
1 \\
\end{array}$ & $\begin{array}{l}18 \\
4 \\
1\end{array}$ & $\begin{array}{c}12 \\
4 \\
1 \\
\end{array}$ & NS & $\begin{array}{c}31 \\
8 \\
1 \\
\end{array}$ & $\begin{array}{c}24 \\
1 \\
0 \\
\end{array}$ & $\begin{array}{l}4 \\
4 \\
2 \\
\end{array}$ & NS \\
\hline $\begin{array}{l}\text { ST.LAB. } \\
\text { ACTIVO } \\
\text { PENSION }\end{array}$ & $\begin{array}{l}11 \\
24\end{array}$ & $\begin{array}{c}4 \\
19\end{array}$ & $\begin{array}{c}6 \\
11\end{array}$ & NS & $\begin{array}{l}12 \\
28\end{array}$ & $\begin{array}{c}4 \\
21\end{array}$ & $\begin{array}{l}5 \\
5\end{array}$ & NS \\
\hline $\begin{array}{l}\text { A. Social } \\
\mathrm{Si} \\
\text { No }\end{array}$ & $\begin{array}{c}1 \\
34\end{array}$ & $\begin{array}{c}3 \\
20\end{array}$ & $\begin{array}{c}4 \\
13\end{array}$ & $\mathrm{P}<0,040$ & $\begin{array}{c}3 \\
37\end{array}$ & $\begin{array}{c}2 \\
23\end{array}$ & $\begin{array}{l}3 \\
7\end{array}$ & NS \\
\hline
\end{tabular}




\section{Tabla 8}

\begin{tabular}{|c|c|c|c|c|c|c|c|c|}
\hline \multicolumn{9}{|c|}{ Correlación ansiedad y depresión con variables proceso dialítico } \\
\hline \multirow{2}{*}{$\begin{array}{l}\text { Variables } \\
\text { sociodem }\end{array}$} & \multicolumn{3}{|c|}{ VARIABLES DEPRESIÓN } & \multirow{2}{*}{$\begin{array}{c}\text { Sign. } \\
\text { estadística }\end{array}$} & \multicolumn{3}{|c|}{ VARIABLES ANSIEDAD } & \multirow{2}{*}{$\begin{array}{c}\text { Sign. } \\
\text { estadística }\end{array}$} \\
\hline & AUSENTE & LEVE & MODERADA & & AUSENTE & LEVE & MODERADA & \\
\hline $\begin{array}{l}\text { TPOenHD } \\
6-33,2 \text { mes } \\
33,2-141 \mathrm{~m} \\
>141 \text { mese }\end{array}$ & $\begin{array}{l}8 \\
8 \\
8\end{array}$ & $\begin{array}{l}11 \\
13 \\
10\end{array}$ & $\begin{array}{l}6 \\
4 \\
7\end{array}$ & NS & $\begin{array}{l}11 \\
13 \\
16\end{array}$ & $\begin{array}{c}10 \\
11 \\
4\end{array}$ & $\begin{array}{l}4 \\
1 \\
5\end{array}$ & NS \\
\hline $\begin{array}{l}\text { TP.Sesión } \\
\leq \text { de } 3,5 \mathrm{~h} . \\
>\text { de } 3,5 \mathrm{~h} .\end{array}$ & $\begin{array}{l}19 \\
16\end{array}$ & $\begin{array}{c}15 \\
8\end{array}$ & $\begin{array}{l}9 \\
8\end{array}$ & NS & $\begin{array}{l}22 \\
18\end{array}$ & $\begin{array}{c}16 \\
9\end{array}$ & $\begin{array}{l}5 \\
5\end{array}$ & NS \\
\hline $\begin{array}{l}\text { TRANSP } \\
\text { PROPIO } \\
\text { COLECT }\end{array}$ & $\begin{array}{l}22 \\
13\end{array}$ & $\begin{array}{l}13 \\
10\end{array}$ & $\begin{array}{c}10 \\
7\end{array}$ & NS & $\begin{array}{l}26 \\
14\end{array}$ & $\begin{array}{l}11 \\
14\end{array}$ & $\begin{array}{l}8 \\
2\end{array}$ & NS \\
\hline $\begin{array}{l}\text { DIURESI } \\
\text { SI } \\
\text { NO }\end{array}$ & $\begin{array}{c}7 \\
28\end{array}$ & $\begin{array}{c}3 \\
23\end{array}$ & $\begin{array}{c}5 \\
12\end{array}$ & NS & $\begin{array}{c}8 \\
32\end{array}$ & $\begin{array}{c}6 \\
19\end{array}$ & $\begin{array}{l}1 \\
9\end{array}$ & NS \\
\hline $\begin{array}{l}\text { PRG. TX. } \\
\text { INCLUID } \\
\text { NO INCL }\end{array}$ & $\begin{array}{l}17 \\
18\end{array}$ & $\begin{array}{c}5 \\
18\end{array}$ & $\begin{array}{c}6 \\
11\end{array}$ & NS & $\begin{array}{l}11 \\
22\end{array}$ & $\begin{array}{c}7 \\
18\end{array}$ & $\begin{array}{l}4 \\
6\end{array}$ & NS \\
\hline $\begin{array}{l}\text { №. FARM } \\
\leq \text { de } 6 \\
>\text { de } 6\end{array}$ & $\begin{array}{l}12 \\
23\end{array}$ & $\begin{array}{l}11 \\
12\end{array}$ & $\begin{array}{c}3 \\
14\end{array}$ & NS & $\begin{array}{l}15 \\
25\end{array}$ & $\begin{array}{l}10 \\
15\end{array}$ & $\begin{array}{l}1 \\
9\end{array}$ & NS \\
\hline $\begin{array}{l}\text { Cum. Tera } \\
\text { BUENO } \\
\text { MALO }\end{array}$ & $\begin{array}{c}26 \\
9\end{array}$ & $\begin{array}{c}17 \\
6\end{array}$ & $\begin{array}{c}15 \\
2\end{array}$ & NS & $\begin{array}{c}31 \\
9\end{array}$ & $\begin{array}{c}19 \\
6\end{array}$ & $\begin{array}{l}8 \\
2\end{array}$ & NS \\
\hline $\begin{array}{l}\text { SOBREPE } \\
<\text { de } 2,5 \mathrm{Kg} \\
\leq \text { de } 2,5 \mathrm{~kg}\end{array}$ & $\begin{array}{l}20 \\
15\end{array}$ & $\begin{array}{l}10 \\
13\end{array}$ & $\begin{array}{l}9 \\
8\end{array}$ & NS & $\begin{array}{l}21 \\
19\end{array}$ & $\begin{array}{l}13 \\
12\end{array}$ & $\begin{array}{l}5 \\
5\end{array}$ & NS \\
\hline
\end{tabular}

En nuestro estudio el análisis estadístico no mostró ninguna asociación significativa entre las variables bioquímicas del proceso dialítico y la ansiedad y la depresión (tabla 9).

\section{Tabla 9}

\begin{tabular}{|c|c|c|c|c|c|c|c|c|c|c|}
\hline \multicolumn{11}{|c|}{ Variables bioquímicas como indicadoras del proceso dialítico } \\
\hline & \multicolumn{2}{|c|}{ Pacientes } & \multicolumn{3}{|c|}{ Sint. Ansiosa } & \multirow[t]{2}{*}{ Sign. Estd. } & \multicolumn{3}{|c|}{ Sint. Depresiva } & \multirow[t]{2}{*}{ Sign. Estd } \\
\hline & & & ente & Leve & Mod. & & Ausente & Leve & Mod. & \\
\hline \multicolumn{11}{|l|}{ KTV como diálisis adecuada } \\
\hline$>1,2$ & 70 & $93,3 \%$ & 31 & 12 & 27 & NS & 36 & 24 & 10 & NS \\
\hline$\leq 1,2$ & 5 & $6,7 \%$ & 3 & 1 & 1 & NS & 4 & 1 & 0 & NS \\
\hline \multicolumn{11}{|l|}{$\mathrm{Hb}$ como índice de calidad } \\
\hline$>$ de $12 \mathrm{~g} / \mathrm{dl}$ & 57 & $76 \%$ & 25 & 17 & 15 & NS & 28 & 23 & 6 & NS \\
\hline$\leq \mathrm{de} 12 \mathrm{~g} / \mathrm{dl}$ & 18 & $24 \%$ & 10 & 6 & 2 & NS & 12 & 2 & 4 & NS \\
\hline \multicolumn{11}{|c|}{ Fósforo como cumpl. terap. } \\
\hline $\begin{array}{l}\leq \text { de } 6 \\
>\text { de } 6\end{array}$ & $\begin{array}{l}53 \\
22\end{array}$ & $29,3 \%$ & $\begin{array}{l}23 \\
18\end{array}$ & $\begin{array}{r}15 \\
8\end{array}$ & $\begin{array}{r}15 \\
2\end{array}$ & NS & $\begin{array}{l}28 \\
12\end{array}$ & $\begin{array}{r}17 \\
8\end{array}$ & $\begin{array}{l}8 \\
2\end{array}$ & NS \\
\hline \multicolumn{11}{|l|}{ Potasio como adhe. Dieta } \\
\hline $\begin{array}{l}\leq \text { de } 6 \mathrm{meq} / \mathrm{L} \\
>\text { de } 6 \mathrm{meq} / \mathrm{L}\end{array}$ & $\begin{array}{l}62 \\
13\end{array}$ & $\begin{array}{l}82,7 \% \\
17,3 \%\end{array}$ & 8 & $\begin{array}{r}21 \\
2\end{array}$ & $\begin{array}{r}14 \\
3\end{array}$ & $\begin{array}{l}\text { NS } \\
\text { NS }\end{array}$ & $\begin{array}{r}31 \\
9\end{array}$ & $\begin{array}{r}24 \\
1\end{array}$ & 3 & $\begin{array}{l}\text { NS } \\
\text { NS }\end{array}$ \\
\hline
\end{tabular}




\section{DISCUSIÓN}

En nuestros centros de diálisis la prevalencia de depresión está presente en un porcentaje bastante elevado de pacientes. El 53,4 \% tenía depresión de algún grado de los cuales el 22,7 \% era grave según la escala de Hamilton. Los síntomas depresivos son inferiores con el Inventario de Beck, pero tenemos que tener en cuenta que éste es un test autoaplicado y el test de Hamilton es heteroaplicado (Tabla 3). La prevalencia de sintomatología depresiva que hemos observado está dentro del rango hallado por otros estudios de pacientes en hemodiálisis $^{4-5-6}$, si bien, existe un amplio abanico de resultados según el método de cribado que se utilice. La prevalencia depresiva detectada en nuestro estudio es superior a la encontrada en pacientes con otra técnica dialítica como puede ser la diálisis peritoneal ${ }^{4-12}$ y muy superior a la detectada en la población general que acude a los centros de Atención Primaria ${ }^{13}$.

La prevalencia de síntomas ansiosos detectada es del $46,6 \%$, con un mayor porcentaje para ansiosos leves $(33,3 \%)$ (Tabla 4), porcentaje similar o levemente superior al hallado en otros estudios ${ }^{2-4}$. Debemos tener presente al interpretar nuestro estudio comparándolo con los de la población general la existencia de síntomas somáticos que pueden enmascarar la clínica ansiosa y depresiva.

La correlación estadística entre los resultados de las escalas utilizadas en este trabajo es muy significativa. Se evidenció un alto nivel de asociación entre los síntomas ansiosos y depresivos medidos por las diferentes escalas (Tabla 5).

Al valorar las variables sociodemográficas observamos que la edad, el sexo femenino, la situación laboral y vivir solo no han resultado factores significativos de ansiedad o depresión (Tabla 7) que son factores muy significativos en trabajos realizados en la población general. Al contrario podemos ver como los hombres y los casados, aun sin ser significativo, tienen índices de ansiedad y depresión superiores a las mujeres y los que viven solos, que son las personas más susceptibles de padecer trastornos afectivos ${ }^{13}$.

Nos ha sorprendido que el escaso porcentaje de pacientes con aislamiento social, que aunque son resultados significativos con la sintomatología depresiva (Tabla 7), es muy inferior a la encontrada en los estudios de la población general que acude a Atención Primaria ${ }^{13-14} y$ la población hospitalizada ${ }^{15}$, donde el aislamiento social es uno de los principales motivos causantes de patología depresiva.
Las variables relacionadas con el proceso dialítico no se han comportado como factores de riesgo relacionadas con la depresión y la ansiedad (Tabla 8). No hemos detectado que llevar más tiempo en diálisis, dializarse más horas, tener menos diuresis, no estar incluido en programa de trasplante o el sobrepeso, favorezca la depresión y la ansiedad. Tampoco observamos ninguna diferencia estadísticamente significativa entre los índices dialíticos bioquímicos y los síntomas ansiosos y depresivos (Tabla 9). En algún otro estudio ${ }^{6}$ estos índices si resultan significativos, pero es debido a que los índices medios de hemoglobina y KTV son inferiores a los nuestros.

La sintomatología ansiosa y depresiva presenta una buena correlación con los resultados obtenidos en las láminas COOP/WONCA (Tabla 6), que nos aportan información acerca de las alteraciones en la capacidad funcional de nuestros pacientes en diálisis y su CVRS. A excepción de la lámina $\mathrm{n}^{\circ} 5$ que indica si han tenido un cambio de salud en los últimos días y la nº 8 que trata del apoyo social, todas las láminas COOP/WONCA se correlacionan bien con los datos de ansiedad y depresión. El COOP TOTAL de nuestros pacientes $(19,08)$ (Tabla 4) es inferior al obtenido por otro estudio con pacientes de Atención Primaria $(20,5)^{14}$, pero con una edad media superior a la de nuestro estudio.

A la vista de los resultados podemos concluir que, según nuestro estudio, existe una elevada prevalencia de sintomatología ansiosa y depresiva en nuestros pacientes de hemodiálisis, con unos resultados dentro del rango hallado en otros estudios. No hemos encontrado factores de riesgo específicos entre las distintas variables demográficas y de diálisis estudiadas que justifiquen la sintomatología ansiosa y depresiva. Factores relacionados con la calidad de vida y capacidad funcional como: un descenso generalizado en la actividad física, la disminución de actividades sociales y la pérdida de energía para mantener sus actividades cotidianas y laborales, sí ha resultado altamente significativa como responsable de la alta incidencia de sintomatología ansiosa y depresiva en nuestros pacientes.

Estos resultados, en general, nos hacen pensar que la alta prevalencia de síntomas ansiosos y depresivos de nuestros pacientes no tiene que ver con factores específicos de riesgo, sino con síntomas físicos como el dolor, o inespecíficos como la salud percibida por el paciente.

Por ello creemos que la enfermería nefrológica y el resto del equipo de la unidad tenemos que abordar al paciente con sospecha de patología ansiosa o depresiva mediante la escucha, la contención y practicando todas las terapias de ayuda y apoyo al enfermo que tenemos a nuestro alcance. 


\section{AGRADECIMIENTOS}

A la Dra. Lorena García Fernández, Beatriz Cantó, Jesús Álvarez, Cristina Carratalá, y en general a todas las compañeras y compañeros de las unidades de diálisis participantes en el estudio por su ayuda y colaboración en la realización de este trabajo.

\section{BIBLIOGRAFÍA}

1. Norman B. Psicología y rehabilitación en: Daugirdas JT, Ing TS. Manual de diálisis. Barcelona: Masson; 1996: 355-358.

2. García-Campayo JJ, Sanz Carrillo C et al. Problemática psicológica en el paciente renal. Nefrología 1992; XII (6): 465-470.

3. Ayuso JL. Trastornos de Angustia. Barcelona: Martinez Roca. 1988.

4. Sacks Carol $\mathrm{R}$ et al. Perception of illness and depression in chronic renal disease. American Journal of Kidney Disease1990; Vol. XV (1): 31-39.

5. Álvarez-Ude F, Fernández-Reyes MJ et al. Síntomas físicos y trastornos emocionales en pacientes en programa de hemodiálisis periódicas. Nefrología 2001; Vol. XXI. (2):191-199.

6. Valderrama FW et al. Mala adherencia a la dieta en hemodiálisis: Papel de los síntomas ansiosos y depresivos. Nefrología 2002; Vol. XXII. (3): 245-251.

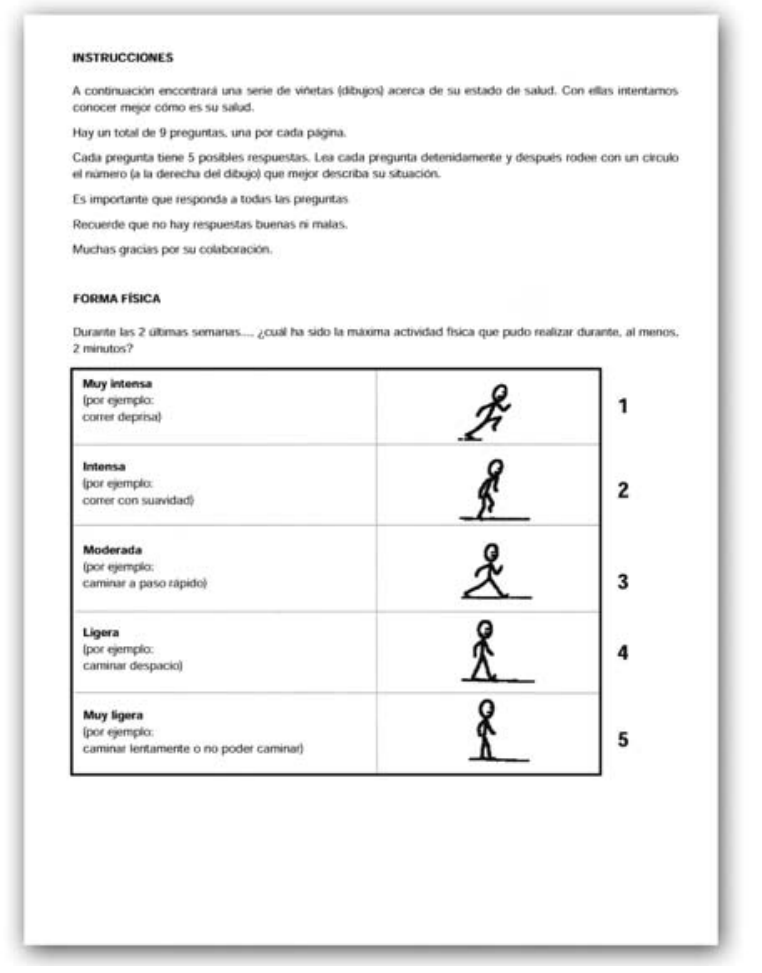

Figura 1. Láminas de COOP/WONCA.
7. Ivorra C, Montiel A, Picó L et al. Apoyo psicosocial a los pacientes en hemodiálisis. Libro comunicaciones XXIV Congreso SEDEN Valencia. 1999: 206-209.

8. Sanz J, Vázquez C. Fiabilidad, validez y datos normativos del inventario para la depresión de Beck. Psicothema. 10, 303-318.

9. Hamilton M. A rating scale for depression. Journal of Neurology Neurosurgery and psichiatry. 1960; 23:56-62.

10. Hamilton M. The assessment of anxiety states by rating. British Journal of Medical Psychology 1959; 32:50-55.

11. Lizán L, Reig A. Adaptación transcultural de una medida de la calidad de vida relacionada con la salud: versión española de las viñetas COOP/WONCA. A. Primaria1999; Vol. 24(2):75-82.

12. Smith MD et al: Diagnosis of depression in patients with end estage renal disease. American journal Mecical 1985; 79:160-166.

13. Aragonés E, Gutierrez M.A et al. Prevalencia, expresión e impacto de los trastornos depresivos en atención primaria. Aten. Primaria 2003; Vol. 31(62):120125.

14. Ferrer E, Rodríguez A. Estudio descriptivo de la patología depresiva en la atención primaria gallega. Anales de Psiquiatría 1999; Vol.15 (2): 68-75.

15. Arias A, Jiménez M. Detección de trastornos depresivos en pacientes hospitalizados. Col. Psiquiatría 1998; Vol. 27 (3): 220-223.

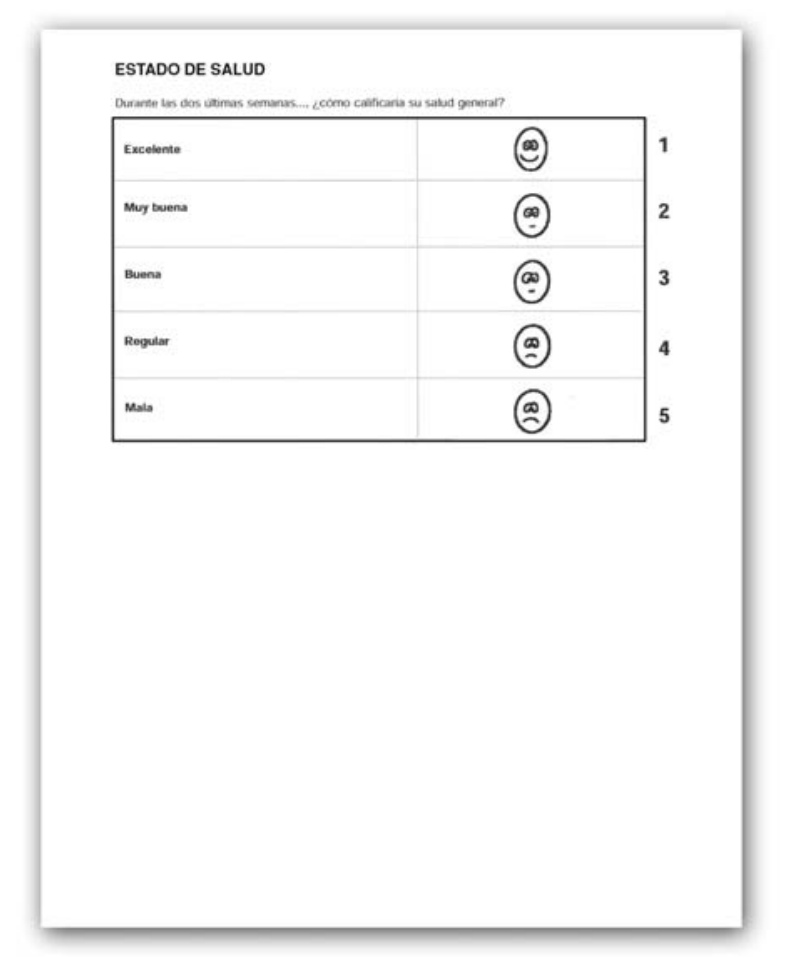

